\title{
Erratum to: T-cell-based vaccination for morphological and functional neuroprotection in a rat model of chronically elevated intraocular pressure
}

\author{
Sharon Bakalash • Gil Ben-Shlomo • Eyal Aloni • \\ Iftach Shaked • Larry Wheeler • Ron Ofri • \\ Michal Schwartz
}

Published online: 11 August 2011

(C) Springer-Verlag 2011

Erratum to: J Mol Med (2005) 83:904-916

DOI: 10.1007/s00109-005-0689-6

The original article unfortunately contained a mistake.

The author's name Gil Ben Shlomo was misspelled. The correct name should be Gil Ben-Shlomo (with a hyphen between "Ben" and "Shlomo") since "Ben-Shlomo" altogether is the last name.

The online version of the original article can be found at http://dx.doi. org/10.1007/s00109-005-0689-6.

S. Bakalash $\cdot$ I. Shaked $\cdot$ M. Schwartz $(\bowtie)$

Department of Neurobiology,

The Weizmann Institute of Science,

76100 Rehovot, Israel

e-mail: michal.schwartz@weizmann.ac.il

G. Ben-Shlomo $・$ R. Ofri

Koret School of Veterinary Medicine,

The Hebrew University of Jerusalem,

Rehovot, Israel

E. Aloni

Department of Ophthalmology,

Kaplan Medical Center,

Rehovot, Israel

L. Wheeler

Department of Biological Sciences,

Allergan Pharmaceuticals,

Irvine, CA, USA 